\title{
Effect of Downstream Flapping Plate on the Flow Field Characteristics behind a Circular Cylinder
}

\author{
Roam Simenthy, Vasudevan Raghavan, Shaligram Tiwari \\ Department of Mechanical Engineering \\ Indian Institute of Technology \\ Madras, Chennai - 600036, India \\ roamsimenthy@gmail.com; raghavan@iitm.ac.in; shaligt@iitm.ac.in
}

\begin{abstract}
Two-dimensional numerical investigations of flow characteristics behind a circular cylinder in the presence of a flapping plate mounted in the wake have been carried out in the present study. The flapping frequency of the plate has been varied in the range from 0.6 to 1.4 times the vortex shedding frequency behind single stationary circular cylinder in the presence of a stationary plate. The gap between the cylinder and the plate is considered to be 1D, 2D and 3D. The maximum amplitude of the tip of the plate has been fixed to be $0.3 \mathrm{D}$ ( $D$ being the diameter of the cylinder) for each of the frequency ratio for fixed value of Reynolds number (Re) equal to 100. The length of the plate has been chosen equal to cylinder diameter. The dynamic effects and the behaviour of vortex shedding behind the cylinders have been studied elaborately. Effect of fixed amplitude and varying frequency ratio of the cylinder on wake flow behaviour has been presented in the form of vorticity contours, lift, drag and Strouhal number (St) plots. The dynamic forces as well as the flow structure behind the plate are found to be modified in the presence of the flapping plate.
\end{abstract}

Keywords: Circular cylinder, flapping plate, gap ratio, vortex shedding, lift and drag.

\section{Introduction}

The effective control of the vortex shedding behind the cylinders becomes an important issue due to their wide range of practical applications like flow past heat exchangers, buildings, suspension bridges, road vehicles to name a few. The vortex shedding the bluff bodies can sometimes be catastrophic due to the fact that the frequency of the vortex shedding from the cylinder matches with the resonance frequency of the structure. The will finally lead to the damage of the structure. From the past itself researchers have considered this as a classic research topic and made a lot of effort to suppress the vortex shedding by flow control, which is meant to control the vortex shedding from the cylinder. Methods of controlling the shedding have been classified as active and passive methods. Among the two types of methods used for controlling the vortex shedding, the active control is found to be more effective. Presence of a wake splitter is one of the effective passive control method used for wake stabilization and drag reduction. The concept of flapping motion is obtained from the motion of a tadpole for its locomotion, from which it is seen that by properly choosing the frequency and the amplitude of flapping the drag of the cylinder can be achieved.

Ali et al. [5] studied the modification of the wake structure of a square cylinder due to the presence of a downstream plate which is placed at varying gap distance between the plate and the cylinder for Reynolds number $(\operatorname{Re})=150$. They identified two flow regimes at critical gap distance of 2.3D and observed that there is no significant effect of the plate on the generation of von Karman vorticies beyond a gap distance of 5.6D. Kwon and Choi [4] observed that when the length of the attached splitter plate is larger than a critical length the vortex formation behind the cylinder vanishes and this critical length is a function of $\mathrm{Re}$. Wu and Shu [2] conducted numerical simulation of laminar flows around a stationary cylinder attached with a flapping plate at its rear side. They showed that the flow structure behind the cylinder and flapping plate is greatly affected by the flapping amplitude, frequency of plate and length of the plate. They observed two vortex interaction modes namely constructive and destructive and explained their effect on the drag force. Bao and Tao [7] numerically analyzed the effect of a stream wise oscillating foil to control the vortex shedding of a circular cylinder. They noticed that the dynamic interaction between the main shear layer and oscillatory boundary layers are responsible for wake stabilization. They identified three flow regimes based on wake stabilization and observed that the increase in oscillation 
amplitude and frequency of the foil augments the wake suppressing effect of the system. Serson et al. [1] performed 2D and 3D simulations for a circular cylinder in the presence of a splitter plate to observe the wake transition in the flow around the cylinder. They noticed that, when the gap between the splitter plate and the cylinder is increased the Strouhal number ( $\mathrm{St}$ ) values showed a discontinuous increase in its value. Hwang et al. [3] studied and explained the flow physics related to the reduction in flow - induced forces happening in the circular cylinder in the presence of a wake splitter plate having a length same as that of the cylinder. They observed a gradual reduction of the flow-induced forces on the cylinder and the subsequent sudden increase over a critical gap ratio between the cylinder and the plate. From their observations, they concluded that the significant reductions of drag and lift fluctuation achieved in unsteady flows using a "detached" splitter plate are strongly related to suppression of the vortex shedding near the circular cylinder. Xiao et al. [6] examined the wake interaction between an undulation NACA0012 foil placed in the wake of a D-sectional cylinder to study the parametric effects such as Re, the relative size of the foil to the cylinder, the foil undulating frequency, the wavelength and the gap between the cylinder and the foil affect the cylinder drag, lift force as well as foil thrust. They showed that when the foil was properly placed at a particular location at the downstream of the cylinder with its chord length equal to the cylinder diameter, suppression of cylinder vortex shedding and an improvement in downstream foil propulsion can be achieved. Qiu et al. [8] experimentally investigated the characteristics of wind loads acting on a circular cylinder with splitter plates attached at the front and/or rear of the cylinder. They observed that the cylinder with a frontal plate has produced a post-critical flow at relatively low Re. Further they noticed that the vortex shedding is suppressed by a splitter plate $(L / D=3)$ in the wake for a semi-cylindrical roof, that can be approximated as a circular cylinder with bilateral plates under similar flow conditions.

\section{Problem Definition}

The computational domain chosen for the present study is shown in Fig. 1. The length and width of the domain are chosen as 40D and 16D, respectively. Air at $1 \mathrm{~atm}$ and $298 \mathrm{~K}$ is considered as the working fluid. The position of the circular cylinder is fixed at 10D from the inlet and the position of the plate is varied such that the gap between the cylinder and the plate, which is measured from the rear side of the cylinder and the front tip of the plate are chosen as 1D, 2D and 3D. The length of the plate is considered to be hinged at the front end and is forced to rotate harmonically in the transverse direction relative to the direction of the incoming flow, such that, $\theta_{z}=\theta \sin \omega t$ where $\theta$ is the angle of rotation about $\mathrm{z}-$ axis and $\omega$ is the frequency of cylinder oscillation. The value of amplitude of flapping $(A)$ chosen for the present study is $0.3 \mathrm{D}$. The frequency ratio is varied from 0.6 to 1.4 . The frequency ratio $\left(f_{r}=f_{e} / f_{o}\right)$ is the ratio of the excitation frequency of the flapper to the frequency of vortex shedding behind the isolated cylinder. All the computations have been carried out for a fixed value of $\operatorname{Re}=100$.

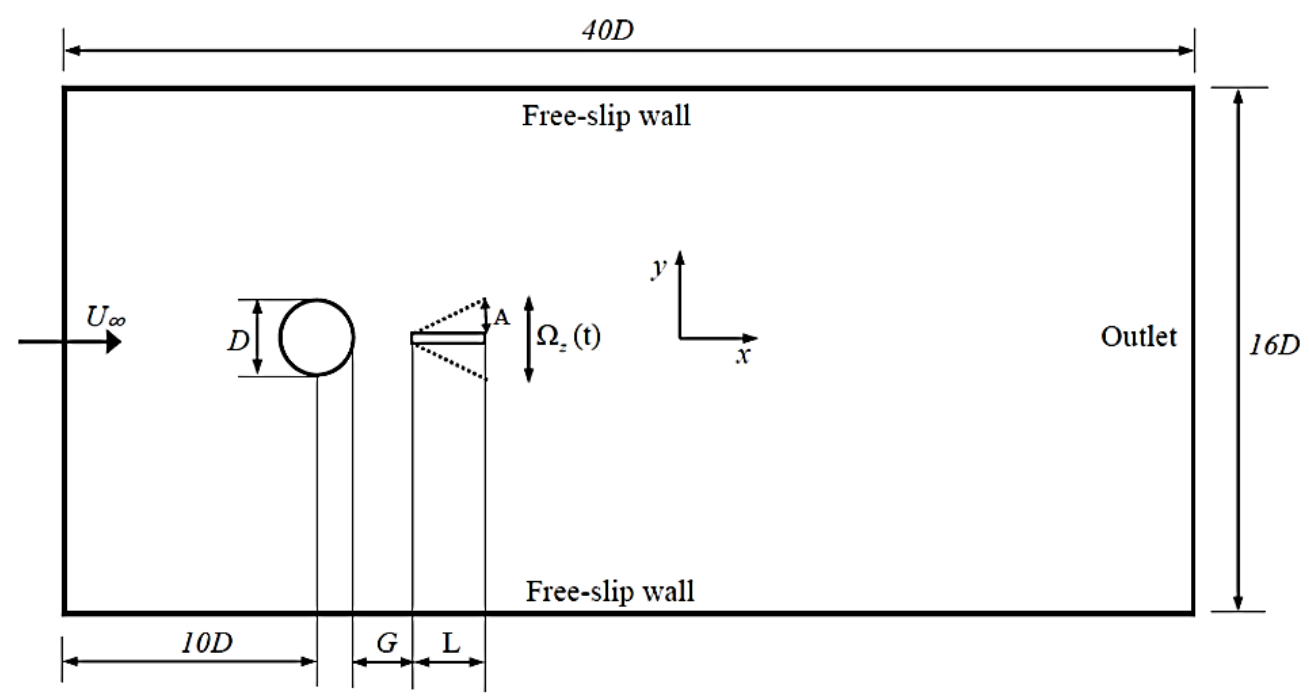

Fig. 1: Schematic of the computational domain. 


\section{Governing Equations and Boundary Conditions}

\subsection{Governing equations}

The governing equations for incompressible and unsteady flow that are required to be solved are those of mass and momentum conservations. For an incompressible flow, the mass conservation equation is used in pressure-velocity coupling for correcting the pressure field using SIMPLE algorithm. The non-dimensional governing equations in tensor form are given as

$$
\begin{gathered}
\frac{\partial u_{i}}{\partial x_{i}}=0 \\
\frac{\partial u_{i}}{\partial t}+\frac{\partial\left(u_{i} u_{j}\right)}{\partial x_{j}}=-\frac{\partial p}{\partial x_{i}}+\frac{1}{\operatorname{Re}}\left(\frac{\partial^{2} u_{i}}{\partial x_{j} \partial x_{j}}\right)
\end{gathered}
$$

where $u_{i}$ is the velocity component along $x_{i}$-coordinate direction and $p$ is the non-dimensional pressure. For a two-dimensional Cartesian coordinate system, Eq. (2) collectively represents the $x$ and $y$-components of the momentum equation for the corresponding velocity components $u$ and $v$, respectively. The flow velocity at the inlet $\left(U_{\infty}\right)$ is considered as the characteristic velocity and the diameter $(D)$ of the circular cylinder is chosen as the characteristic length. Accordingly, the flow Reynolds number is given by

$$
\operatorname{Re}=\frac{\rho U_{\infty} D}{\mu}
$$

Here $\rho$ is the density of the fluid at standard conditions of the free stream and $\mu$ is its dynamic viscosity. The nondimensionalized static pressure at any location is given as,

$$
p=\frac{P-P_{\infty}}{\rho U_{\infty}^{2}}
$$

\subsection{Boundary conditions}

Inlet: Uniform velocity in $\mathrm{x}$-direction, $u=1.0$ and $v=0$

Outlet: Pressure outlet, $p=0$

Top and bottom walls: Free-slip condition and impermeable, $\frac{\partial u}{\partial y}=0 ; \quad v=0$

Circular cylinder surface: no-slip and impermeable boundary, i.e., $u=v=0$

Plate: $u=0 ; \Omega_{z}=\theta \omega \cos \omega t$, where $\Omega_{z}$, the rotational velocity of the plate about z-axis , $\theta$ is the angular amplitude of the plate measured from the mean position.

\section{Grid Generation and Numerical Technique}

\subsection{Grid generation}

Hybrid grid have been used to divide the entire flow domain into discrete finite volumes. The grid generation has been done in such a way that the grids are near the cylinder surface and the region outside the flapping plate is quadrilateral type. A region with triangular grid is generated in order to accommodate the motion of the plate. A grid independence study has been performed to optimize the number of grids such that best accuracy can be achieved with optimum 
computational time. The results from the grid independence study have been presented in Table 1 . The variations of mean drag, RMS lift coefficient and Strouhal number obtained for an isolated circular cylinder using different grids are also shown in Table-1. Finally, a grid mesh having 160 grid points on the cylinder surface is chosen for all the computations because no significant variation is found in the computed values of above quantities with further refinement. The validation of the results obtained for the cylinder in the presence of plate has been presented in the following section.

Table 1: Grid independence study for isolated stationary cylinder.

\begin{tabular}{|c|c|c|c|}
\hline $\begin{array}{c}\text { No. of grid } \\
\text { points on the } \\
\text { cylinder }\end{array}$ & $\begin{array}{c}\text { RMS Lift } \\
\text { coefficient } \\
\left(\mathrm{C}_{1, \text { rms }}\right)\end{array}$ & $\begin{array}{c}\text { Mean drag } \\
\text { coefficient } \\
\left(\mathrm{C}_{\mathrm{d}, \text { mean }}\right)\end{array}$ & $\begin{array}{c}\text { Strouhal } \\
\text { number } \\
(\mathrm{St})\end{array}$ \\
\hline 80 & 0.259 & 1.495 & 0.1806 \\
\hline 120 & 0.263 & 1.499 & 0.1797 \\
\hline 160 & 0.262 & 1.499 & 0.1798 \\
\hline 200 & 0.262 & 1.498 & 0.1798 \\
\hline
\end{tabular}

\subsection{Numerical technique}

As mentioned earlier, the governing equations are solved using the commercial software ANSYS Fluent 14.0 based on the finite volume method. The software uses SIMPLE (Semi Implicit Method for Pressure Linked Equations) algorithm is used for pressure-velocity coupling. For discretization unsteady term, first order accurate implicit time marching scheme has been used. Convective terms are discretized using second order upwind scheme, while the diffusive terms are discretized using the central difference scheme. The harmonic rotational motion of the plate is incorporated with the help of a User Defined Function (UDF) and by using the dynamic meshing capability of ANSYS Fluent 14.0, which takes care of the re-meshing when the plate is displaced at each time step.

\section{Results and Discussion}

Computations have been carried out for flow past a circular cylinder with a flapping plate mounted at the downstream. The spacing between the cylinder and flapper as well as amplitude and frequency of oscillation of the flapper are varied. The front end of the plate is hinged and the angle of rotation of the plate is chosen such that the rear tip of the plate reaches a maximum amplitude of $0.3 \mathrm{D}$ from the mean position, i.e. the centreline of the cylinder in horizontal position. The validation of the computed results has been first carried out for a single stationary circular cylinder followed by the plate when it is stationary. Using the validated model, lift and drag fluctuation as well as vorticity contours are simulated and presented in the following subsections.

\subsection{Validation}

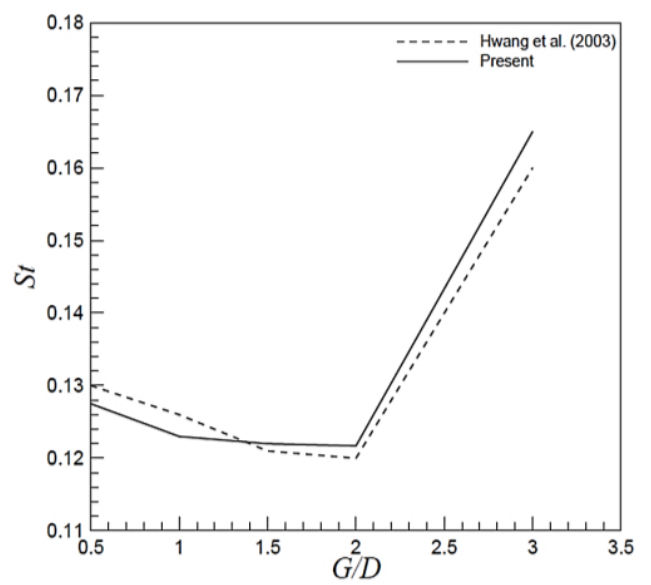

Fig. 2: Validation of predicted value of Strouhal number at different gap spacing for a stationary plate with result from literature. 
Figure 2 shows the variation of St with different gap spacing between the cylinder and the plate, when the plate is stationary at a fixed value of $\operatorname{Re}=100$. The results are validated with that of Hwang et al. (2003) and shows a good agreement.

\subsection{Flow characteristics behind circular cylinders due to flapping motion of the plate}

Variation of mean drag coefficient $\left(C_{d, \text { mean }}\right)$ with frequency ratio $\left(f_{r}\right)$ is shown in Fig. 3 . Solid lines represent the drag values for the cylinder when the plate is flapping and dashed lines represent the corresponding values when the plate is kept stationary. For $G / D=1$ the drag values show a gradual increment up to $f_{r}=1.0$ and then starts decreasing. It is noted that the presence of the plate is obvious in this spacing and the vortex shedding frequency of the cylinder is same as that of the flapping frequency of the plate for all values of $f_{r}$. Similarly for $G / D=2$, the interference effect of the flapper is visible for $f_{r}$ values higher than 0.8 and shows increment in drag values up to $f_{r}=1.0$ and then starts decreasing. For $f_{r}=0.6$ and 0.8 , the drag values are same as that of stationary values. Further when the gap between the cylinder and plate is increased such that $G / D=3$, the drag values are comparatively higher for $f_{r}=0.6$ and 0.8 and then there is a sudden drop in the value for $f_{r}=0.9$, which is lower than the stationary value. Then the value again rises up to $f_{r}=1.1$ and then starts to decrease such that at $f_{r}=1.4$ the drag coefficient drops to a value lower than its stationary configuration. Thus for all the values of flapping frequency and the gap ratios considered, drag reduction is observed at $G / D=3$ for $f_{r}=0.9$ and 1.4.

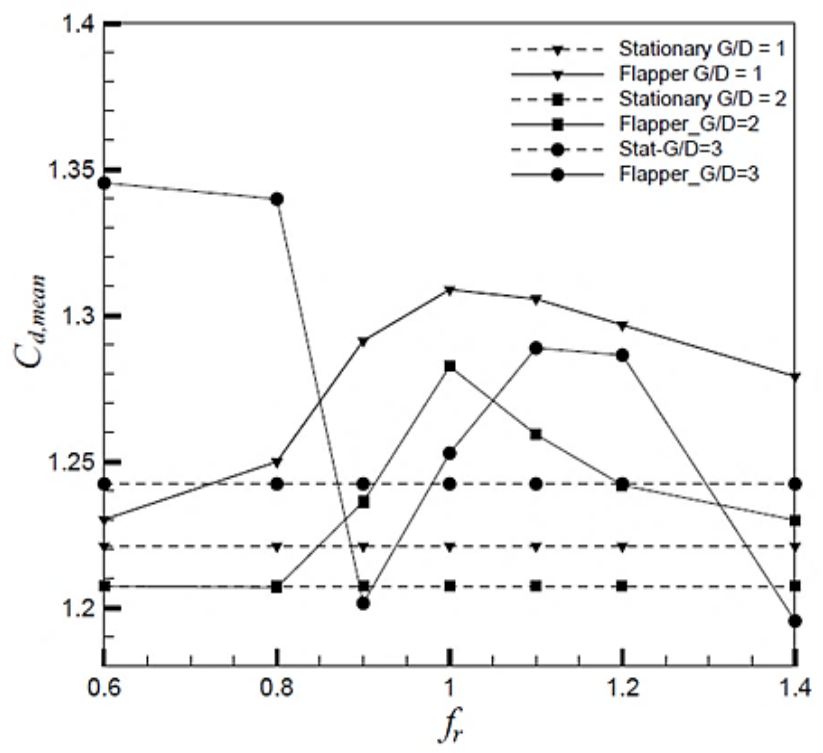

(a)

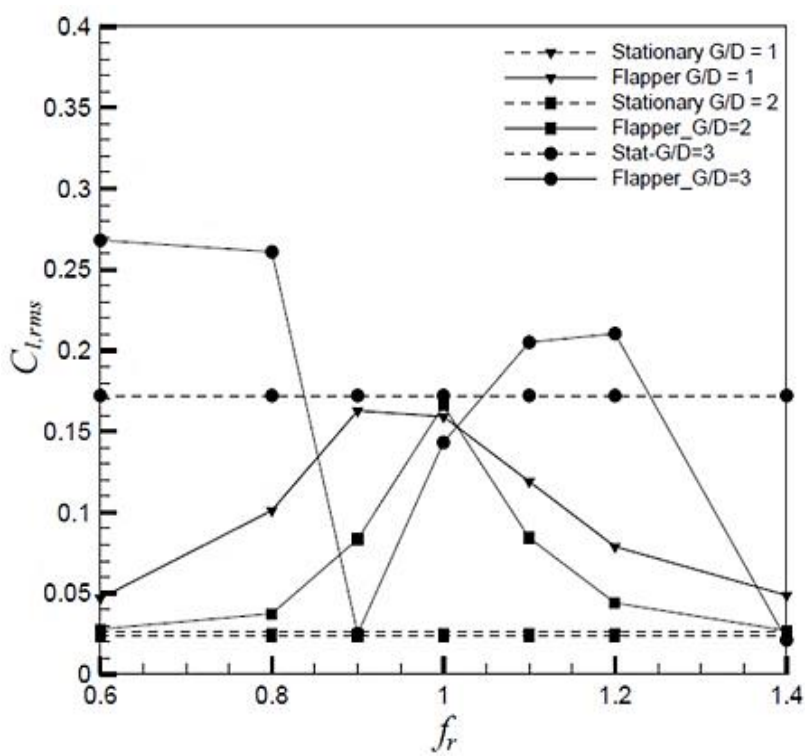

(b)

Fig. 3: Variation of (a) mean drag coefficient (b) RMS lift coefficient with frequency ratio and for different values of spacing.

Figure 3 (b) shows the variation of RMS lift coefficient $\left(C_{1, r m s}\right)$ with $f_{r}$. The variation of $C_{1, r m s}$ also follows the same trend as that of $C_{d, \text { mean }}$. For $G / D=1$, the $C_{1, r m s}$ value is always higher than that of the stationary value for all values of $f_{r}$, which implies that the effect of flapping of the plate tends to create more fluctuation in the vertical forces acting on the cylinder. When the gap between the plate and the cylinder is increased to a value $G / D=2$, for lower values of $f_{r}$ such as $f_{r}=0.6$ and 0.8 and for higher values of $f_{r}$ like $f_{r}=1.2$ and $1.4, C_{1, r m s}$ values are closer to their stationary values as depicted in the figure. The maximum values of $C_{1, r m s}$ occurs at $f_{r}=0.9$ for $G / D=1$ and $f_{r}=1$ for $G / D=2$. Further when the spacing is increased to $G / D=3, C_{1, r m s}$ values are considerably reduced for $f_{r}=0.9$ and 1.4 as similar to that 
observed for $C_{d, \text { mean }}$. The dynamic forces acting on the cylinders can be considerably reduced when the plate is placed at $G / D=3$ and oscillated at $f_{r}=0.9$ and 1.4 at $\mathrm{A}=0.3$. Thus it can be affirmed that there is a significant effect of amplitude and frequency of oscillation of the plate on the flow - induced forces acting on the cylinder.

Figure 4 shows the comparison of Strouhal number $(\mathrm{St})$ values for the circular cylinder due to the presence of flapper. It is clearly seen that at spacing values of $1 \mathrm{D}$ and $2 \mathrm{D}$, the vortex shedding behind the cylinder is influenced by the cylinder such that the shedding frequency matches with the flapping frequency of the plate. On the other hand, at $G / D=3$, for all the values of $f_{r}$ except for $f_{r}=0.9$ and 1.4 , the shedding frequency $\left(f_{s}\right)$ of the cylinder is different from that of the flapping frequency and is found to be a constant value. But for the above two cases, $f_{s}$ matches with the excitation frequency of the flapper $\left(f_{e}\right)$ as observed in the case of $G / D=2$ and 3 .

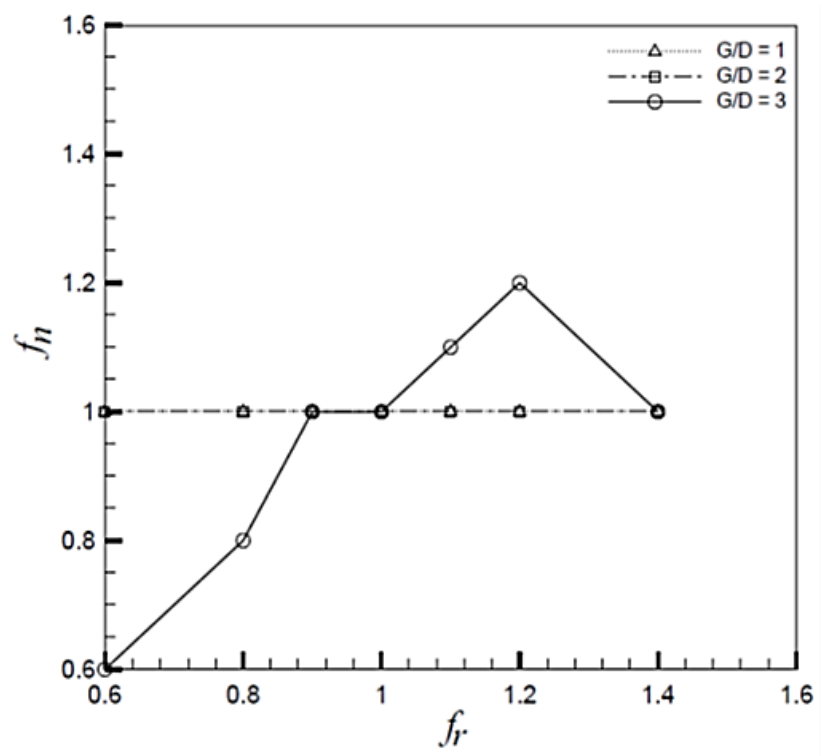

Fig. 4: Comparison of predicted value of St at different values of spacing for circular cylinder in the presence of flapping plate.

Instantaneous vorticity contours for $G / D=3$ have been presented in Fig. 5. As seen from the plots of $C_{d, \text { mean }}$ and $C_{1, r m s}$, the values are lower than their corresponding stationary values for $f_{r}=0.9$ and 1.4 as shown in Fig.5(b) and 5(d). In both the cases, vortex shedding behind the cylinder is suppressed due to the flapping motion of the plate. Where as in the other two cases, i.e. for $f_{r}=0.6$ and 1.1, the vortex shedding takes place in the gap between the cylinder and the plate. Thus the reduction of flow-induced forces acting on the cylinder may be attributed to the suppression of vortex shedding due to the flapping motion of the plate. It is also noted that reduction in the forces is more predominant in the case of $f_{r}=1.4$ than $f_{r}=0.9$ because the drag reduction is more in the former case. For the other cases, the vortices shed from the cylinder are able to interact with each other and this in turn produces comparatively larger fluctuating forces in the horizontal and vertical directions. This is due to the fact that at lower values of $f_{r}$, the vortices shed from the cylinder are comparatively smaller in size and the interaction between the shear layers becomes much stronger. At higher values of $f_{r}$, the sizes of the vortices are slightly higher and they almost hit the front side of the plate. This decreases the interaction of vortices in the gap of the cylinder and the plate and thus results into reduced magnitudes of forces. From the analysis of St data, it is observed that the regular vortex shedding frequency also gets affected due to oscillation of the plate. 


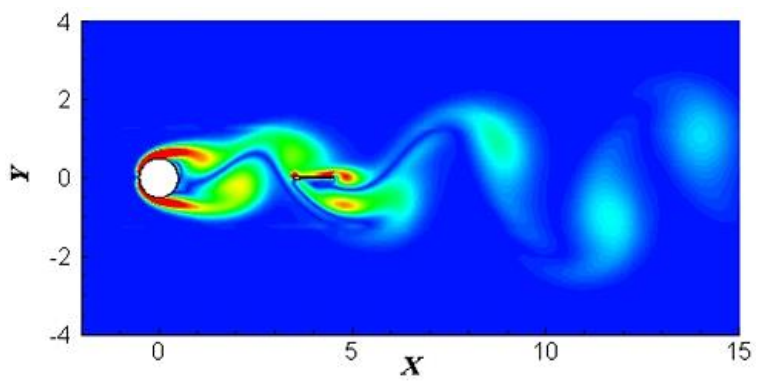

(a)

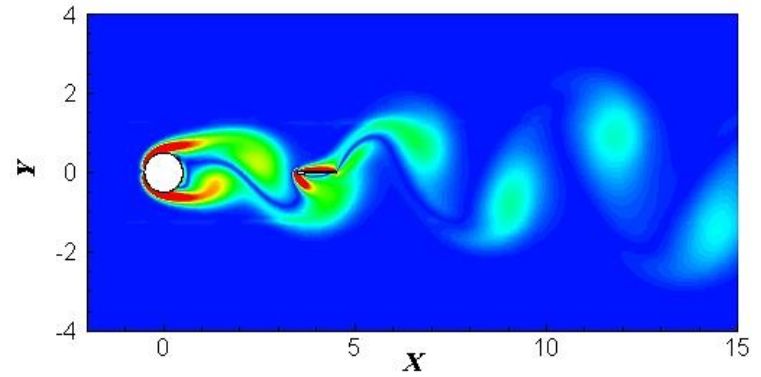

(c)

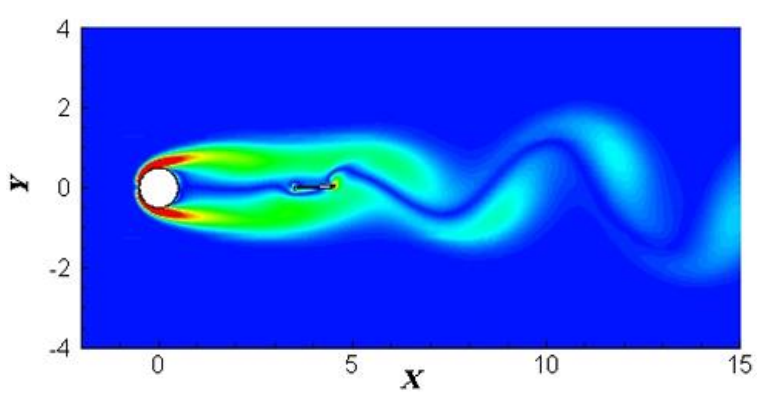

(b)

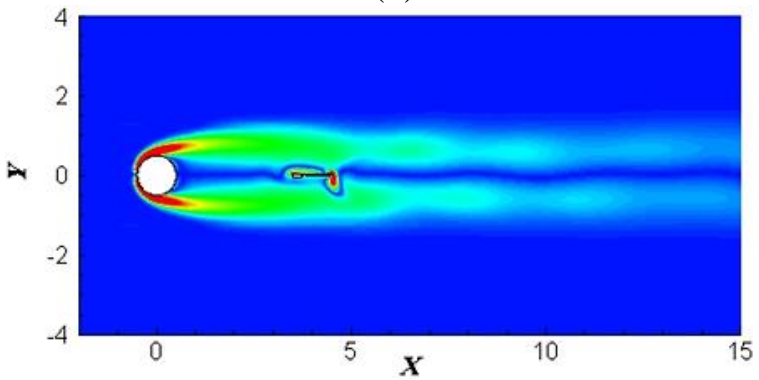

(d)

Fig. 5: Instantaneous vorticity contours for circular cylinder in the presence of the flapping plate at its mean position for $\mathrm{G} / \mathrm{D}=3$ and (a) $f_{r}=0.6$ (b) $f_{r}=0.9$ (c) $f_{r}=1.1$ (d) $f_{r}=1.4$.

\section{Conclusion}

The effect of downstream flapping plate on a circular cylinder has been investigated for different values of spacing between cylinder and plate and varying frequency ratios for plate oscillation, at a fixed value of Re. The fluid dynamic forces acting on the cylinder are considerably reduced than the corresponding value for stationary case when the plate is placed at $G / D=3$ and oscillated at $f_{r}=0.9$ and 1.4. The vortex shedding from the cylinder is also found to get suppressed at these values of $f_{r}$. Thus there is a significant effect of amplitude and frequency of oscillation of the plate on the flow-induced forces acting on the cylinder. From the St data it is observed that the regular vortex shedding frequency is affected due to the oscillation of the plate. Moreover, at $G / D=3$, the shedding frequency and the flapping frequency of the plate are different, whereas both the frequencies become same at all other value of spacing.

\section{References}

[1] D. Serson, J. R. Meneghini, B. S. Carmo, E. V. Volpe, and R. S. Gioria., "Wake transition in the flow around a circular cylinder with a splitter plate," J. Fluid Mech., vol. 755, pp. 582-602, 2014.

[2] J. Wu and C. Shu, "Numerical study of flow characteristics behind a stationary circular cylinder with a flapping plate," Phys. Fluids., vol. 23, no. 073601, 2011.

[3] J. Y. Hwang, K. S. Yang, and S. H. Sun, "Reduction of flow-induced forces on a circular cylinder using a detached splitter plate," Phys. Fluids., vol. 15, no. 8, 2003.

[4] K. Kwon and H. Choi, "Control of laminar vortex shedding behind a circular cylinder using splitter plates," Phys. Fluids, vol. 8, no. 479, 1996.

[5] M. S. M Ali, C. J. Doolan, and V. Wheatley, "Low Reynolds number flow over a square cylinder with a detached flat plate," Int. J. Heat Fluid Flow, vol. 36, pp. 133-141, 2012.

[6] Q. Xiao, W. Liu, and J. Hu, "Parametric study on a cylinder drag reduction using downstream undulating foil," Eur. $J$. Mech B-Fluid, vol. 36, pp. 48-62, 2012.

[7] Y. Bao and J. Tao, "Active control of a cylinder wake flow by using a streamwise oscillating foil," Phys. Fluids, vol. 25, no. 053601, 2013.

[8] Y. Qiu, Y. Sun, Y. Wu, and Y. Tamura, "Effects of splitter plates and Reynolds number on the aerodynamic loads acting on a circular cylinder," J. Wind Eng. Ind. Aerodyn., vol. 127, pp. 40-50, 2014. 